\title{
The Variable Rate Intravenous Insulin Infusion Protocol
}

Benjamin Collard, Jonathan Sturgeon, Natasha Patel, Shabbar Asharia

St Georges Healthcare NHS Trust

\begin{abstract}
Insulin use among inpatients is high and associated with severe and regular medication errors. An initial baseline audit showed a wide variation in the prescription of intravenous insulin within the trust. These included variation in the choice of fluid prescribed, electrolyte levels not consistently checked, handwritten illegible prescriptions, and varying parameters set for adjustment of the prescription.

A Variable Rate Intravenous Insulin Infusion protocol (VRIII)) was introduced to standardize intravenous insulin prescription throughout the trust by all members of the clinical team. We looked at and measured uptake and effects of the VRIII protocol in improving standardization of insulin prescription for inpatients on insulin at St George's NHS trust. The protocol was uploaded to the intranet to allow access 24 hours a day and the staff educated about it.
\end{abstract}

The VRIII protocol was routinely used successfully throughout the trust. Any initial problems were addressed through education of clinical staff. The protocol has shown decreased prescribing and administrative errors, whilst demonstrating good glucose and electrolyte control.

Use of a standardized protocol helps reduce medication errors and demonstrates good glycaemic control. Regular and continued education of clinical staff is necessary to maintain its efficacy.

\section{Problem}

Intravenous insulin prescription has traditionally varied widely between different clinicians and Hospitals. We conducted a baseline audit to assess the standardisation of intravenous insulin prescription in our trust. We did this by reviewing inpatients receiving intravenous insulin at St George's NHS trust. We compared the patient notes, reviewed the fluids prescribed, glucose levels, biochemistry checked and the types of scales used.

We found 20 different intravenous scales used to prescribe intravenous insulin. We found different fluids prescribed, with varying degrees of legibility (Figure 1) Different parameters were set on each scale as to when the prescription should be adjusted or senior help should be enlisted. Only half of the patients had had their potassium checked daily, and three patients were found to be hypokalaemic (Graph 1).

There were four issues identified with the current intravenous insulin prescriptions:

1. The insulin infusion rates were not adjusted according to physiological requirements, for example surgery, pain, stress etc.

2. The insulin infusion rates did not take in to account the patients normal insulin regimen. The same scale was used for type 1 and type 2 diabetics and no consideration was given for any endogenous insulin secretion.

3. The definition of hypoglycaemia varied.

4. The potassium and sodium levels were frequently not recorded.

\section{Background}

In the United Kingdom (UK), the prevalence of diabetes mellitus types 1 and 2 in adults is now $4.45 \%$ of the general population (1), and the prevalence of diabetes among inpatients is as high as $15 \%$. (2) This number is only likely to get higher as the prevalence and incidence of type 2 diabetes have increased in the UK over the past decade. In addition inpatient population with diabetes tend to be older than other patients with median age 75 years compared to 67 years for all inpatients.

People with diabetes also have a substantially longer length of stay with median length of stay at 8 nights compared to 5 nights for all inpatients. (2) Of those with diabetes mellitus around a quarter require injected insulin as part of their treatment, and in hospitals this percentage is around $40 \%(2,3)$. As insulin has a narrow therapeutic window, this routine use can present a challenge to ensure the correct dose is titrated to blood sugar levels, and given at the correct time.

The National Diabetes Inpatient Audit (NDIA) 2010 collected data on over 12,000 diabetic inpatients from UK hospitals and discovered that errors in drug management had occurred in $20 \%$ of patients (2). This frequency of error is cause for concern, as errors with insulin are twice as likely to cause harm as errors for other prescribed drugs, and has resulted in six deaths and twelve cases of severe harm in the UK (4-6).

The recent National Diabetes Audit cited the risk of death for people with type 1 and type 2 diabetes being 2.6 and 1.6 times higher than that of the general population respectively. (7) Evidence from NICESUGAR (Normoglycaemia in Intensive Care Evaluation Survival 
Using Glucose Algorithm Regulation) study recommends that all critically ill patients within a critical care unit should be treated with a revised, less aggressive variable rate intravenous insulin infusion (VRIII) algorithm (previously known as a sliding scale). (8) This risk of hypoglycaemia among all inpatients on insulin remains high irrespective of their critical status.

This study was an assessment of uptake of the VRIII protocol (Figure 1) among medical inpatients receiving intravenous insulin at St George's NHS trust.

\section{Baseline Measurement}

We conducted a baseline audit to assess the standardisation of intravenous insulin prescription in our trust. We did this by reviewing inpatients receiving intravenous insulin at St George's NHS trust. We compared the patient notes, reviewed the fluids prescribed, glucose levels, biochemistry checked and the types of scales used (Figure 1). We found 20 different intravenous scales used to prescribe intravenous insulin. We found different fluids prescribed, with varying degrees of legibility. Different parameters were set on each scale as to when the prescription should be adjusted or senior help should be enlisted. Only half of the patients had had their potassium checked daily, and three patients were found to be hypokalaemic (Graph 1).

There were four issues identified with the current intravenous insulin prescriptions:

1. The insulin infusion rates were not adjusted according to physiological requirements, for example surgery, pain, stress etc.

2. The insulin infusion rates did not take in to account the patients normal insulin regimen. The same scale was used for type 1 and type 2 diabetics and no consideration was given for any endogenous insulin secretion.

3. The definition of hypoglycaemia varied.

4. The potassium and sodium levels were frequently not recorded.

See supplementary file: ds2452.doc - "VRIII Graph 1 Figure 1"

\section{Design}

The perceived problems highlighted the need for a standardised variable rate intravenous insulin infusion (VRIII) protocol. There were several considerations which had to be taken in to account. Firstly it had to be safe, ensuring that it minimized the risk of hypoor hyperglycaemia. This meant there had to be an available choice of sliding scale appropriate to the patient, as well as frequent monitoring of glucose levels. For doctors, it had to be easy to understand and prescribe, and the prescription should only require a signature. It should be easily implemented to reduce administration errors. Finally it should encourage the switch to subcutaneous insulin as soon as clinically appropriate, and be accessible throughout the hospital.
The rationale behind the introduction of new VRIII was one of patient safety as well as providing structure for medical staff. Standardizing prescriptions allows staff of all backgrounds to easily notice errors, as well as speed up prescribing. It dictates the appropriate fluids to give and advises the correct monitoring of potassium and glucose levels. The infusion levels can also easily be tailored to the patient's circumstances by choosing one of several scales, and a target blood glucose level. This should ideally result in improved blood sugar and electrolyte control.

This project examined the inpatient use of intravenous insulin in St George's NHS trust. Intravenous insulin should be limited to those who are unable to take anything by mouth, or to those with diarrhoea and vomiting. This project evaluated the introduction of a variable rate intravenous insulin infusion (VRIII) protocol, and audited its effect on clinical practice. We decided to audit immediately after the protocols introduction and the audit again 6 months later after education regarding the usage of the protocol.

\section{Strategy}

We introduced this VRIII protocol throughout St Georges Hospital NHS Trust. We proceeded to educate the clinical staff regarding its administration. Thereafter we audited its usage initially over a two month period and monitored the accuracy of application. All inpatients at St Georges NHS Trust receiving intravenous insulin were included in the audit.

After making initial changes the protocol was re-audited after six months. This was done again by reviewing all patients within St George's NHS Trust receiving intravenous insulin during a two month period.

\section{Results}

An early discovery from the audits was that some patients were still being given intravenous insulin when it was no longer necessary (Graph 2). This problem was due to inexperience in the prescribing of insulin and we sought to rectify this through the educating of both junior doctors and ward nurses. This was no longer a problem by our final audit. There was also a decline in the incidence of hypoglycaemia to $5 \%$ and hypokalaemia to $5 \%$. Finally it demonstrated an improved awareness of when more senior support was required for advice.

From our study we found that the Variable Rate Intravenous Insulin Infusion protocol had been well accepted throughout the trust. Through staff education via lectures and one to one sessions on individual wards, confidence was gained using the new regime. All members of the clinical team found the standardised approach easier, quicker and safer to use. The uploading of our protocol to the intranet ensured it was accessible 24 hours a day throughout the trust.

Our initial audit raised concerns with the prescription and administration of intravenous insulin. We discovered over 20 different regimes being used which varied in fluid prescription, 
usage of potassium, hypoglycaemia classification and timing of conversion to subcutaneous insulin. Our latest audit showed $100 \%$ of doctors prescribed intravenous insulin appropriately and no patient safety errors documented (Graph 3). The same fluid was prescribed in $100 \%$ of cases, and in all cases potassium was checked at least once. The VRIII was never prescribed inappropriately and it was always attached to the drug chart and used with a dedicated cannula. The sodium and glucose levels were monitored throughout, and the intravenous insulin prescription adjusted as needed.

See supplementary file: ds2453.doc - "VRIII All Graphs"

\section{Lessons and Limitations}

The project was initially limited by the high turnover of clinical staff. Doctors in particular change jobs and specialties frequently. Also locum and bank staff are common in most NHS trusts. We overcame this problem by educating on our staff induction days and uploading out protocol to the hospital intranet. This enabled it to be accessed 24 hours a day. In our final audit we achieved 100\% success of the protocol been prescribed.

\section{Conclusion}

From our exercise we found that the VRIII is now used successfully throughout the trust and is widely available through the hospital intranet. Initial concerns have been addressed through education of clinical staff. The protocol has shown decreased prescribing and administrative errors, whilst demonstrating good glucose and electrolyte control.

In the future it is imperative we continue to educate clinical staff regarding its appropriate usage. In 6 months time we will reaudit to ensure the new protocol is still used correctly and throughout the trust by all clinical members of the team.

\section{References}

1. Diabetes UK. Diabetes in the UK 2012. Available at: http://www.diabetes.org.uk/Documents/Reports/Diabetes-inthe-UK-2012.pdf

2. NHS Diabetes. 2010 Available from: http://www.diabetes.nhs.uk/document.php?o=334

3. U.S. Department of Health and Human Services, Centers for Disease Control and Prevention, 2011. National Diabetes Fact Sheet: national estimates and general information on diabetes and prediabetes in the United States. Available from: http://diabetes.niddk.nih.gov/dm/pubs/statistics/DM Statistic s_508.pdf

4. Lamont T, Cousins D, Hillson R, Bischler A, Terblanche M. Safer administration of insulin: summary of a safety report from the National Patient Safety Agency. BMJ. 2010; 341: 5269.

5. National Patient Safety Agency. NPSA/2011/PSA003: Patient Safety Alert. 2011 March 30.
6. Cheung N, Chipps D. Sliding scale insulin: will the false idol finally fall? Internal Medicine Journal. 2010; 40: 662-4.

7. NHS Information Centre (2011) National Diabetes Audit Mortality Analysis 2007-2008. The NHS Information Centre for health and social care, Leeds. Available at: http://bit.ly/rDi1/r.pdf.

8. NICE-SUGAR Study Investigators et al. Intensive versus conventional glucose control in critically ill patients. N Engl J Med 2009;360:1283-97.

\section{Declaration of interests}

Nothing to declare

\section{Acknowledgements}

No acknowledgements 